\title{
Chromosome-Level Assembly of the Chinese Seabass (Lateolabrax maculatus) Genome
}

\author{
Baohua Chen ${ }^{1,2}$, Yun $\mathrm{Li}^{3}$, Wenzhu Peng ${ }^{1,4}$, Zhixiong Zhou ${ }^{1,4}$, Yue Shi ${ }^{1,4}$, Fei $\mathrm{Pu}^{1,2}$, \\ Xuan Luo ${ }^{1,4}$, Lin Chen ${ }^{1,4}$ and Peng $X^{2,4,5 *}$ \\ ${ }^{1}$ State Key Laboratory of Marine Environmental Science, College of Ocean and Earth Sciences, Xiamen University, Xiamen, \\ China, ${ }^{2}$ Shenzhen Research Institute of Xiamen University, Shenzhen, China, ${ }^{3}$ The Key Laboratory of Mariculture, Ministry of \\ Education, Ocean University of China, Qingdao, China, ${ }^{4}$ State-Province Joint Engineer Laboratory of Marine Bioproducts \\ and Technology, College of Ocean and Earth Sciences, Xiamen University, Xiamen, China, ${ }^{5}$ Laboratory for Marine Biology \\ and Biotechnology, Qingdao National Laboratory for Marine Science and Technology, Qingdao, China
}

Keywords: Lateolabrax maculatus, seabass, genome assembly, Hi-C, teleost genome

\section{INTRODUCTION}

OPEN ACCESS

Edited by:

Hans Cheng,

U.S. National Poultry Research Center (ARS-USDA), United States

Reviewed by:

Geoff Waldbieser

Warmwater Aquaculture Research Unit (ARS-USDA), United States

Yniv Palti,

Cool and Cold Water Aquaculture Research (ARS-USDA), United States

*Correspondence:

Peng Xu

xupeng77@xmu.edu.cn

Specialty section:

This article was submitted to

Livestock Genomics,

a section of the journal

Frontiers in Genetics

Received: 03 December 2018 Accepted: 12 March 2019

Published: 04 April 2019

Citation:

Chen B, Li Y, Peng W, Zhou Z, Shi Y, Pu F, Luo $X$, Chen L and Xu P (2019) Chromosome-Level Assembly of the

Chinese Seabass (Lateolabrax maculatus) Genome.

Front. Genet. 10:275

doi: 10.3389/fgene.2019.00275
The Chinese seabass (Lateolabrax maculatus), inhabiting in inshore rocky reefs and estuaries with a broad adaptability of salinity, is an euryhaline teleost fish native to the margin seas of the Northwest Pacific Ocean (Figure 1A). The Chinese seabass belongs to genus Lateolabrax that was first described as a geographic population of Japanese seabass Lateolabrax japonicas. It was recently re-described as independent species from L. japonicas, based on the differentiated characters of morphological traits and molecular phylogenies (Liu et al., 2006; An et al., 2014). In contrast with geographically restricted L. japonicas, L. maculatus is broadly and continuously distributed along the coasts of China and Indo-China Peninsula (Yokogawa and Seki, 1995). The north most of wildlife habitats of L. maculatus is latitude $41^{\circ}$ north in temperate Bohai Gulf and the south most reaches at least $20^{\circ}$ north in tropical Beibu Gulf, between which the sea surface temperature difference frequently reaches $18^{\circ} \mathrm{C}$ in winter. The very different environments of $L$. maculatus that live in Bohai Gulf and Beibu Gulf make them divergent in genetic structures and phenotypes, such as life history, behaviors, and breeding season etc., providing us with a feasible fish model for population genetic studies in continual marginal sea (Zhao et al., 2018). In addition, L. maculatus is recognized as one of the most important mariculture fish in China, which contributes over 120,000 tons of annual production. Recently, a reference genome of L. maculatus derived from in the northern population in Bohai Gulf had been reported (Shao et al., 2018). Herein, we report a chromosome-level genome assembly of L. maculatus from the southern population in the subtropical region, which provides an important resource not only for basic ecological and population genetic studies but also for the upcoming breeding program of Chinese seabass.

\section{DATA}

A whole genome shotgun (WGS) strategy was employed in this project. After removal of redundant and low-quality reads, there are a total of $112.76 \mathrm{~Gb}$ (188.87X) clean WGS reads, including 49.63, 31.89, 19.69, and $11.66 \mathrm{~Gb}$ reads from $250 \mathrm{bp}, 2 \mathrm{Kbp}, 5 \mathrm{Kbp}$, and $10 \mathrm{Kbp}$ libraries, respectively, obtained for genome size estimation, de novo contig assembly, primary scaffolding. High-through chromosome conformation capture (Hi-C) sequencing were performed for chromosome-level scaffolds construction. A total of $159.54 \mathrm{~Gb}$ pair-end $\mathrm{Hi}-\mathrm{C}$ reads were generated with an average sequencing coverage of 267.06X (Table 1). 
A

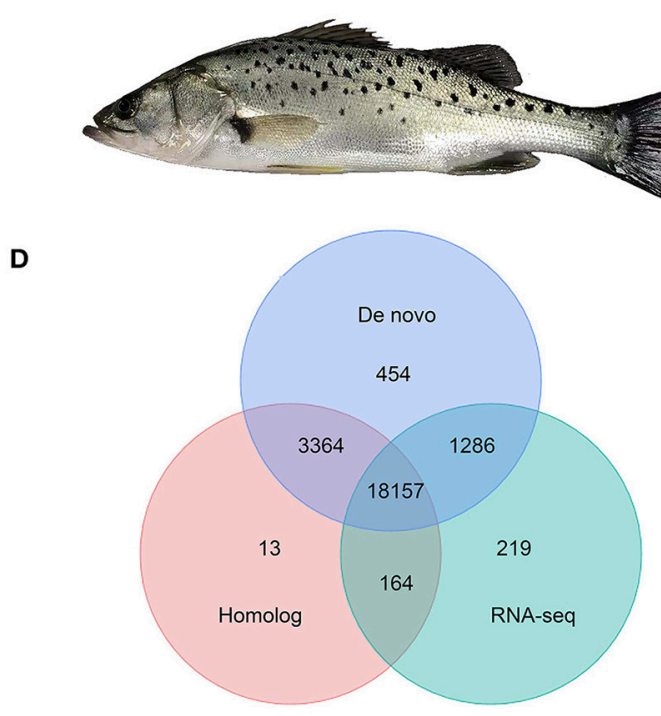

E

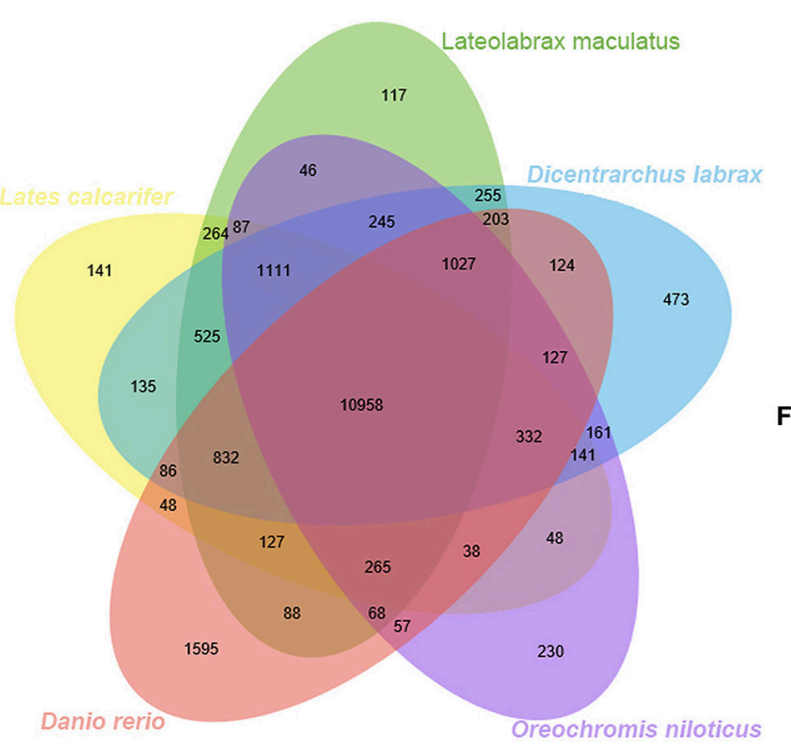

B
C
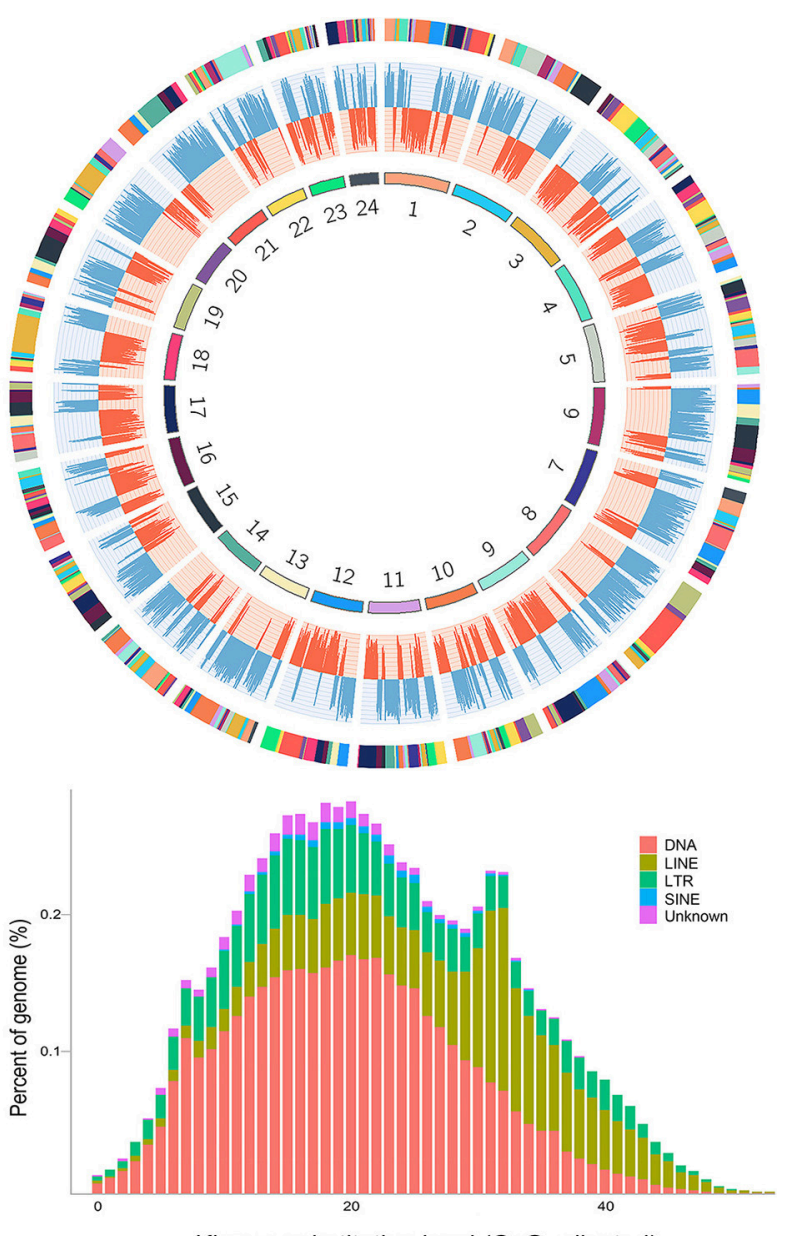

Kimura substitution level ( $\mathrm{CpG}$ adjusted)

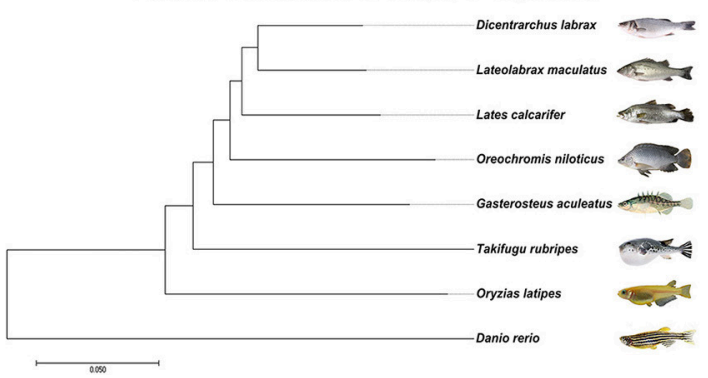

FIGURE 1 | Characteristics of Lateolabrax maculatus genome assembly. (A) A picture showing about Chinese seabass, L. maculatus. (B) A circos plot of 24 chromosomes in L. maculatus genome, the tracks from inside to outside are: 24 chromosomes, gene abundance of the positive strand (red), gene abundance of the negative strand (blue), and scaffolds which comprised the chromosome (adjacent contigs on a chromosome are painted in different colors). (C) Divergence distribution of TEs in L. maculatus Genome. (D) A venn diagram indicating the number of genes predicted by three different approaches. (E) A venn diagram showing orthologous gene families across five fish genomes. (F) Evolutionary relationships among eight species.

Based on all reads mentioned above, we de novo assembled the draft L. maculatus genome with a size of $597.39 \mathrm{Mb}$ containing 1,639 scaffolds. And the contig N50 size was $182.31 \mathrm{~kb}$ and the scaffold N50 size was $2.79 \mathrm{Mb}$. After integrating the scaffolds with Hi-C map, we finally obtained 24 chromosomes constructed from 419 scaffolds (25.56\% of all scaffolds) with a total length of $586.03 \mathrm{Mb}(98.10 \%$ of the total length of all scaffolds) (Table 1 and Figure 1B). Our new reference genome has been significantly improved compared with the previous reference genome of the northern population, which presents contig N50 length of $31 \mathrm{~Kb}$, scaffold N50 length of $1,040 \mathrm{~Kb}$, and chromosome integration rate of $77.68 \%$ (Shao et al., 2018).

A total of $105.5 \mathrm{Mb}(\sim 17.66 \%$ of $L$. maculatus genome) were identified as repetitive elements in the L. maculatus genome, including $6.09 \%$ of DNA transposons, $4.99 \%$ of long interspersed nuclear elements (LINEs), and $2.31 \%$ of long terminal repeats (LTRs) (Figure 1C). 
TABLE 1 | Summary of the L. maculatus genome assembly and annotation.

\begin{tabular}{|c|c|c|}
\hline & Bases (Gb) & Depth \\
\hline \multicolumn{3}{|l|}{ SEQUENCING } \\
\hline Illumina reads (short-insert size) & 49.63 & $83.01 X$ \\
\hline Illumina reads (long-insert size) & 63.24 & 105.86X \\
\hline $\mathrm{Hi}-\mathrm{C}$ reads & 159.54 & $267.06 \mathrm{X}$ \\
\hline \multicolumn{3}{|c|}{ GENOME ASSEMBLY AND CHROMOSOMES CONSTRUCTION } \\
\hline Contig N50 size (kbp) & \multicolumn{2}{|c|}{182.31} \\
\hline Contig number (>100 bp) & \multicolumn{2}{|c|}{8719} \\
\hline Scaffold N50 size (Mbp) & \multicolumn{2}{|c|}{2.79} \\
\hline Scaffold number (>100 bp) & \multicolumn{2}{|c|}{1639} \\
\hline Scaffold total length (Mbp) & \multicolumn{2}{|c|}{597.39} \\
\hline Number of chromosomes & \multicolumn{2}{|c|}{24} \\
\hline $\begin{array}{l}\text { Number of placed scaffolds on } \\
\text { chromosomes }\end{array}$ & \multicolumn{2}{|c|}{419} \\
\hline Total length of chromosomes (Mbp) & \multicolumn{2}{|c|}{586.03} \\
\hline Integration efficiency of $\mathrm{Hi}-\mathrm{C}$ map (\%) & \multicolumn{2}{|c|}{98.10} \\
\hline \multicolumn{3}{|c|}{ GENOME PREDICTION AND ANNOTATION } \\
\hline Protein-coding gene number & \multicolumn{2}{|c|}{23,657} \\
\hline Mean transcript length (bp) & \multicolumn{2}{|c|}{$13,943.84$} \\
\hline Mean exons per gene & \multicolumn{2}{|c|}{9.75} \\
\hline Proportion of assembled CEGs (\%) & \multicolumn{2}{|c|}{94.76} \\
\hline $\begin{array}{l}\text { Proportion of complete BUSCO } \\
\text { orthologs (\%) }\end{array}$ & \multicolumn{2}{|c|}{97.03} \\
\hline
\end{tabular}

Gene structure prediction identified 23,657 protein-coding genes, of which 22,509 genes can be annotated against at least one database (Figure 1D), and 1,734 candidate non-coding RNAs, including 676, 644, 99, and 315 miRNA, tRNA, rRNA, and snRNA genes, respectively (Table 1).

To evaluate the accuracy of the genome assembly, we mapped Illumina short reads that were used for genome assembly and identified 904,102 heterozygous SNPs and 12,050 false homozygous SNPs, respectively, accounting to 0.1557 and $0.0004 \%$ of the reference genome. The homozygous SNPs were false because they refer to the SNPs that only retained one alternative allele in the Illumina short reads (homozygotes for short reads data), which was different from the reference genome. The low rate of false SNPs suggests the high accuracy of the genome assembly.

The completeness and connectivity of this assembly were accessed using both Core Eukaryotic Genes Mapping Approach (CEGMA) and Benchmarking Universal Single-Copy Orthologs (BUSCO) approaches. Two hundred and thirty-five Core Eukaryotic Genes (CEGs) out of the complete set of 248 CGEs (94.76\%) were covered by the assembly and 818 out of 843 searched BUSCOs (97.03\%) had been completely assembled in the draft genome, suggesting a high level of completeness and connectivity of the de novo assembly (Table 1).

For better use of this dataset, the evolutionary position of $L$. maculatus was accessed based on single-copy genes of $L$. maculatus and seven related species (T. rubripes, G. aculeatus, $O$. latipes, D. rerio, O. niloticus, L. calcarifer, and D. labrax).

The protein sequences were downloaded from the Ensembl Core database (release 90). After removing the protein sequences shorter than 50 amino acids, the set of 245,644 consensus protein sequences of the seven teleost and Chinese seabass L. maculatus was used to construct gene families. As a result, a total of 20,788 OrthoMCL families were built (Figure 1E) and 667 single-copy ortholog protein families in a 1:1:1 manner from all eight teleost species were used for phylogenetic analysis (Figure 1F).

\section{MATERIALS AND METHODS}

\section{Sample Collection, Library Construction, and Sequencing}

A wild adult female Chinese seabass was collected in the Xiamen Bay, Fujian, China and used to collect blood sample. The total length and body weight of this fish were $524.6 \mathrm{~g}$ and $34.5 \mathrm{~cm}$, respectively. Total RNA and DNA extraction were performed for whole genome sequencing and whole transcriptome sequencing following our previous studies (Jiang et al., 2014; Peng et al., 2016). Four whole-genome shotgun sequencing libraries were prepared with various insert sizes ranging from $250 \mathrm{bp}$ to 10 Kbp (250 bp, $2 \mathrm{Kbp}, 5 \mathrm{Kbp}, 10 \mathrm{Kbp}$ ). The $250 \mathrm{bp}$ pair-end library was constructed for de novo contig assembly and the other three mate-pair libraries were constructed for scaffolding contigs. Before sequencing, a quality control step was performed on Agilent 2100 Bioanalyzer (Agilent Technologies, Santa Clara, CA, USA) by evaluating the distribution of fragment length. Then libraries were sequenced using the Illumina HiSeq2500 platform with a read length of $2 \times 150 \mathrm{bp}$.

High-through chromosome conformation capture (Hi-C) were performed parallelly to the Illumina sequencing. DNA samples, collected from muscle tissue, were snap frozen using liquid nitrogen for $30 \mathrm{~min}$ and then stored at $-80^{\circ} \mathrm{C}$ until DNA extraction. Firstly, the DNA was fixed by formaldehyde to maintain the conformation. Then it was digested by MboI restriction enzyme and repaired by biotinylated residues to form blunt-end fragments. After in-situ ligation of these fragments, DNA was reverse-crosslinked and purified. Before sequencing, end repair, adaptor ligation, and polymerase chain reaction were successively performed. At last, the well-prepared Hi-C libraries were sequenced using Illumina Hiseq 2500 platform with a read length of $2 \times 150 \mathrm{bp}$.

\section{Genome Assembly}

All low-quality Illumina read pairs were filtered out if any read of the pair complies with following criteria: containing adaptor sequences; the proportion of uncertain bases (represented by "N") exceed 10\%; the proportion of low-quality base $(Q<5)$ exceed $50 \%$. After strict filtration clean, all Illumina reads were used to generate 17-mers with a window-sliding-like method. Obviously, there were $4^{17}$ kinds of different 17-mers. After calculation of depth distribution of these 17-mers using Jellyfish (Marcais and Kingsford, 2011) (version 2.2.5), we can estimate the genome size using Lander/Waterman's equations:

$$
\begin{aligned}
\mathrm{C}_{\text {base }} & =\mathrm{C}_{17-\text { mer }} \times \mathrm{L} /(\mathrm{L}-17+1) \\
\mathrm{G}_{\text {est }} & =\mathrm{N}_{17-\text { mer }} / \mathrm{C}_{17-\text { mer }}=\mathrm{N}_{\text {base }} / \mathrm{C}_{\text {base }}
\end{aligned}
$$


In these equations, $\mathrm{L}$ was the length of reads (150 for Illumina reads), $\mathrm{N}_{\text {base }}$ and $\mathrm{N}_{17-\text { mer }}$ were counts of bases and 17-mers; $\mathrm{C}_{\text {base }}$ and $\mathrm{C}_{\mathrm{k}-\text { mer }}$ were expectations of coverage depth of bases and 17-mers; estimated genome size was represented by $\mathrm{G}_{\text {est }}$. The genome size of Latiolabrax maculatus was then estimated to contain $641.02 \mathrm{Mb}$, which is similar to Asian seabass (Lates calcarifer, 668.5 Mb) (Vij et al., 2016) and European seabass (Dicentrarchus labrax, 675 Mb) (Tine et al., 2014).

For de novo genome assembly, high quality reads from the short-insert library (250 bp) were collected and assembled using SOAPdenovo2 (Luo et al., 2012) with optimized parameters to build initial contigs. Long-insert reads were then mapped onto the de novo assembled contigs for scaffolding (2, 5, and $10 \mathrm{Kbp}$, in turn). The GapCloser (Luo et al., 2012) was then used to close the gaps in scaffolds using the pair-end reads, of which one end uniquely mapped to a contig and another was located within a gap.

In order to obtain chromosome-level genome assembly, $\mathrm{Hi}-\mathrm{C}$ reads were filtered in the same way as short-insert library reads and subsequently mapped to de novo assembled scaffolds to construct contacts among scaffolds using bwa (Li and Durbin, 2009) (version 0.7.17) with default parameters. Obtained BAM files containing Hi-C read-pairs linking messages were processed by another round of filtering, in which reads located further than $500 \mathrm{bp}$ from the nearest restriction enzyme site were removed. Then LACHESIS (Korbel and Lee, 2013) (version 2e27abb) was used to chromosome-level scaffolding by clustering, ordering and orientating the de novo genome assemblies based on genomics proximity messages between $\mathrm{Hi}-\mathrm{C}$ reads pairs. In these steps, all parameters were set as default except that CLUSTER_N, CLUSTER_MIN_RE_SITES and ORDER_MIN_N_RES_IN_SHREDS were set as 24, 80, and 10 separately. Note that the parameter CLUSTER_N was used to specify the number of chromosomes.

Both karyotype analysis and recently published genome assembly for Chinese seabass (spotted seabass) indicated that the number of chromosomes of this species is 24 (Sola et al., 1993; Shao et al., 2018). Besides, genetics maps of two species in Perciformes, European seabass (Dicentrarchus labrax) and Asian Seabass (Lates calcarifer), both contain 24 linkage groups (Wang et al., 2011; Tine et al., 2014).

\section{Repetitive Elements Characterization}

We employed two approaches to detect repeat sequences in L. maculatus genome. Firstly, we used Tandem Repeats Finder (Benson, 1999) (version 4.04), Piler (Edgar and Myers, 2005) (version 1.0), LTR_FINDER (Xu and Wang, 2007) (version 1.0.2), RepeatModeler (Tarailo-Graovac and Chen, 2009) (version 1.04), and RepeatScout (Price et al., 2005) (version 1.0.2) synchronously to detect various kinds of repeat sequences in $L$. maculatus genome. The results were then combined as a single de novo repeat sequence library by Uclust (Edgar, 2010) (version 1.2.22q). Subsequently, the whole library was annotated using RepeatMasker (Tarailo-Graovac and Chen, 2009) (version 3.2.9) based on Repbase TE (Jurka et al., 2005) (version 14.04) to discriminate between known and novel transposable elements (TEs). In another approach, generated genome sequences were mapped on Repbase TE (Jurka et al., 2005) (version 14.04) using RepeatProteinMask (Tarailo-Graovac and Chen, 2009) (version 3.2.2), a perl script included in RepeatMasker, to detect TE proteins in L. maculatues genome. The results of two approaches were combined and then the redundancy was removed to obtain a final Repetitive elements set.

\section{Gene Structure Prediction}

To access a fully annotated $L$. maculatus genome, three different approaches were employed to predict protein-coding genes. $A b$ intio gene prediction was performed on repeat-masked L. maculatus genome assembly using Augustus (Stanke and Morgenstern, 2005) (version 2.5.5), GlimmerHMM (Majoros et al., 2004) (version 3.0.1), SNAP (Korf, 2004) (version 1.0), Geneid (Parra et al., 2000) (version 1.4.4), and GenScan (Burge and Karlin, 1997) (version 1.0). Furthermore, homology-based prediction was performed using downloaded protein sequences of closely related teleost including Takifugu rubripes (Aparicio et al., 2002), Gasterosteus aculeatus (Jones et al., 2012), Oryzias latipes (Kasahara et al., 2007), Danio rerio (Howe et al., 2013), Oreochromis niloticus (Brawand et al., 2014), Lates calcarifer (Vij et al., 2016), Larimichthys crocea (Ao et al., 2015), and Cynoglossus semilaevis (Chen et al., 2014). Subsequently, these protein sequences were mapped onto the generated assembly using blat (Kent, 2002) (version 35) with $e$-value $\leq 1 \mathrm{e}-5$. GeneWise (Birney et al., 2004) (version 2.2.0) was employed to align the homologs in $L$. maculatus genome against the other species for gene structure prediction. In addition, we also applied transcriptome-based prediction by using RNAseq datasets of a pooled cDNA library of 12 tissues from the fish which was used for whole genome sequencing. The RNA-seq reads were mapped onto the genome assembly using TopHat (Trapnell et al., 2009) (version 1.2) software. The structures of all transcribed genes were predicted by Cufflinks (Trapnell et al., 2010) (version 2.2.1) with default parameters. The predicted gene sets generated from three approaches were then integrated to a non-redundant gene set using EvidenceModeler (Haas et al., 2008) (version 1.1.0). PASA (Haas et al., 2003) (version 2.0.2) was then used to annotate the gene structures. Aiming at identifying candidate non-coding RNA (ncRNA) genes, we aligned repeat-masked genome sequences against Rfam database (Burge et al., 2013) (version 11.0) using BLASTN to search homologs.

\section{Functional Annotation of Genes}

Genes identified by structure prediction were subsequently functionally annotated by BLAST searches against the NCBI $\mathrm{nr}$ and SwissProt protein databases. Unidirectional best-hit of each L. maculatus gene was assigned as its homolog after discarding those with $E$-value $<1 \times 10^{-5}$ by alignments. Gene ontology (GO) annotations of genes were assigned using the InterProScan program (version 5.26) (Quevillon et al., 2005). KEGG annotation was performed against KEGG database, the KEGG Automatic Annotation Server (KAAS) (Moriya et al., 2007). 


\section{The Completeness and Accuracy of the Assembly}

The completeness and accuracy of the assembly were further assessed. We mapped Illumina short reads that were used for genome assembly using bwa (Li and Durbin, 2009) (version 0.7.17-r1188). Subsequently, BAM files containing mapping message were then piled up using samtools ( $\mathrm{Li}$ et al., 2009) (version 1.8) to identify SNPs using thresholds of read depth $>10$ and quality score $>20$. Then, the assembly completeness was evaluated by Core Eukaryotic Genes Mapping Approach (CEGMA) (Parra et al., 2007) (version 2.3) and Benchmarking Universal Single-Copy Orthologs (BUSCO) (Simao et al., 2015) software (version 1.22) using vertebratespecific database (vertebrata_odb9).

\section{Ortholog Analysis}

Single-copy genes in L. maculatus and related species were identified based on gene families constructed from protein sequences of all species employing OrthoMCL (Li et al., 2003) and BLASTP software with default parameters. As there are no corresponding CDS sequences of Asian seabass proteins used in gene family analysis provided, the Asian seabass transcripts were translated into proteins using ORFinder (version 0.4.1). Single-copy ortholog proteins were aligned by MUSCLE (Edgar, 2004) (version 3.8.31). Subsequently, all obtained alignments were converted to their corresponding coding DNA sequences using an internal python script.

\section{REFERENCES}

An, H. S., Kim, H. Y., Kim, J. B., Chang, D. S., Park, K. D., Lee, J. W., et al. (2014). Genetic characterization of hatchery populations of Korean spotted sea bass (Lateolabrax maculatus) using multiplex polymerase chain reaction assays. Genet. Mol. Res. 13, 6701-6715. doi: 10.4238/2014.August.28.14

Ao, J. Q., Mu, Y. N., Xiang, L. X., Fan, D. D., Feng, M. J., Zhang, S. C., et al. (2015). Genome sequencing of the perciform fish Larimichthys crocea provides insights into molecular and genetic mechanisms of stress adaptation. PLoS Genet. 11:e1005118. doi: 10.1371/journal.pgen.1005118

Aparicio, S., Chapman, J., Stupka, E., Putnam, N., Chia, J. M., Dehal, P., et al. (2002). Whole-genome shotgun assembly and analysis of the genome of Fugu rubripes. Science. 297, 1301-1310. doi: 10.1126/science.1072104

Benson, G. (1999). Tandem repeats finder: a program to analyze DNA sequences. Nucleic Acids Res. 27, 573-580. doi: 10.1093/nar/27.2.573

Birney, E., Clamp, M., and Durbin, R. (2004). GeneWise and genomewise. Genome Res. 14, 988-995. doi: 10.1101/gr.1865504

Brawand, D., Wagner, C. E., Li, Y. I., Malinsky, M., Keller, I., Fan, S. H., et al. (2014). The genomic substrate for adaptive radiation in African cichlid fish. Nature. 513, 375-381. doi: 10.1038/nature13726

Burge, C., and Karlin, S. (1997). Prediction of complete gene structures in human genomic DNA. J. Mol. Biol. 268, 78-94. doi: 10.1006/jmbi.1997.0951

Burge, S. W., Daub, J., Eberhardt, R., Tate, J., Barquist, L., Nawrocki, E. P., et al. (2013). Rfam 11.0: 10 years of RNA families. Nucleic Acids Res. 41, D226-D232. doi: 10.1093/nar/gks1005

Chen, S. L., Zhang, G. J., Shao, C. W., Huang, Q. F., Liu, G., Zhang, P., et al. (2014). Whole-genome sequence of a flatfish provides insights into ZW sex chromosome evolution and adaptation to a benthic lifestyle. Nat. Genet. 46, 253-260. doi: 10.1038/ng.2890

Edgar, R. C. (2004). MUSCLE: multiple sequence alignment with high accuracy and high throughput. Nucleic Acids Res. 32, 1792-1797. doi: $10.1093 /$ nar/gkh340
A combined "supergene" was constructed from all the translated coding DNA alignments for minimum evolution (ME) phylogenetic tree construction using MEGA (Kumar et al., 2016) (Version 7.0.26).

\section{ETHICS STATEMENT}

This study was approved by the Animal Care and Use Committee, College of Ocean and Earth Sciences, Xiamen University. The methods were carried out in accordance with approved guidelines.

\section{AUTHOR CONTRIBUTIONS}

PX conceived the study. BC, WP, and YL performed bioinformatics analysis. YL, FP, YS, and XL collected samples. $\mathrm{ZZ}$ and LC extracted DNA and RNA. ZZ and YS performed the quality control. BC and PX wrote the manuscript. All authors read and approved the final manuscript.

\section{FUNDING}

This work was supported by the Knowledge Innovation Program of Shenzhen City (Fundamental Research, Free Exploration, No. JCYJ20170818142601870) and Fundamental Research Funds for the Central Universities, Xiamen University (No. 20720160110).

Edgar, R. C. (2010). Search and clustering orders of magnitude faster than BLAST. Bioinformatics 26, 2460-2461. doi: 10.1093/bioinformatics/ btq461

Edgar, R. C., and Myers, E. W. (2005). PILER: identification and classification of genomic repeats. Bioinformatics. 21, I152-I158. doi: 10.1093/bioinformatics/bti1003

Haas, B. J., Delcher, A. L., Mount, S. M., Wortman, J. R., Smith, R. K., Hannick, L. I., et al. (2003). Improving the Arabidopsis genome annotation using maximal transcript alignment assemblies. Nucleic Acids Res. 31, 5654-5666. doi: 10.1093/nar/gkg770

Haas, B. J., Salzberg, S. L., Zhu, W., Pertea, M., Allen, J. E., Orvis, J., et al. (2008). Automated eukaryotic gene structure annotation using EVidenceModeler and the program to assemble spliced alignments. Genome Biol. 9:R7. doi: 10.1186/gb-2008-9-1-r7

Howe, K., Clark, M. D., Torroja, C. F., Torrance, J., Berthelot, C., Muffato, M., et al. (2013). The zebrafish reference genome sequence and its relationship to the human genome. Nature. 496, 498-503. doi: 10.1038/nature 12111

Jiang, Y., Zhang, S., Xu, J., Feng, J., Mahboob, S., Al-Ghanim, K. A., et al. (2014). Comparative transcriptome analysis reveals the genetic basis of skin color variation in common carp. PLoS ONE. 9:e108200. doi: 10.1371/journal.pone.0108200

Jones, F. C., Grabherr, M. G., Chan, Y. F., Russell, P., Mauceli, E., Johnson, J., et al. (2012). The genomic basis of adaptive evolution in threespine sticklebacks. Nature. 484, 55-61. doi: 10.1038/nature10944

Jurka, J., Kapitonov, V. V., Pavlicek, A., Klonowski, P., Kohany, O., and Walichiewicz, J. (2005). Repbase update, a database of eukaryotic repetitive elements. Cytogenet. Genome Res. 110, 462-467. doi: 10.1159/0000 84979

Kasahara, M., Naruse, K., Sasaki, S., Nakatani, Y., Qu, W., Ahsan, B., et al. (2007). The medaka draft genome and insights into vertebrate genome evolution. Nature. 447, 714-719. doi: 10.1038/nature05846 
Kent, W. J. (2002). BLAT - The BLAST-like alignment tool. Genome Res. 12, 656-664. doi: 10.1101/gr.229202

Korbel, J. O., and Lee, C. (2013). Genome assembly and haplotyping with Hi-C. Nat. Biotechnol. 31, 1099-1101. doi: 10.1038/nbt.2764

Korf, I. (2004). Gene finding in novel genomes. BMC Bioinformatics. 5:59. doi: 10.1186/1471-2105-5-59

Kumar, S., Stecher, G., and Tamura, K. (2016). MEGA7: Molecular Evolutionary Genetics Analysis Version 7.0 for bigger datasets. Mol. Biol. Evol. 33, 1870-1874. doi: 10.1093/molbev/msw054

Li, H., and Durbin, R. (2009). Fast and accurate short read alignment with Burrows-Wheeler transform. Bioinformatics. 25, 1754-1760. doi: 10.1093/bioinformatics/btp324

Li, H., Handsaker, B., Wysoker, A., Fennell, T., Ruan, J., Homer, N., et al. (2009). The Sequence Alignment/Map format and SAMtools. Bioinformatics 25, 20782079. doi: 10.1093/bioinformatics/btp352

Li, L., Stoeckert, C. J. Jr., and Roos, D. S. (2003). OrthoMCL: identification of ortholog groups for eukaryotic genomes. Genome Res. 13, 2178-2189. doi: 10.1101/gr.1224503

Liu, J. X., Gao, T. X., Yokogawa, K., and Zhang, Y. P. (2006). Differential population structuring and demographic history of two closely related fish species, Japanese sea bass (Lateolabrax japonicus) and spotted sea bass (Lateolabrax maculatus) in Northwestern Pacific. Mol. Phylogenet. Evol. 39, 799-811. doi: 10.1016/j.ympev.2006.01.009

Luo, R. B., Liu, B. H., Xie, Y. L., Li, Z. Y., Huang, W. H., Yuan, J. Y., et al. (2012). SOAPdenovo2: an empirically improved memory-efficient short-read de novo assembler. Gigascience. 1:18. doi: 10.1186/2047-217X-1-18

Majoros, W. H., Pertea, M., and Salzberg, S. L. (2004). TigrScan and GlimmerHMM: two open source ab initio eukaryotic genefinders. Bioinformatics. 20, 2878-2879. doi: 10.1093/bioinformatics/ bth315

Marcais, G., and Kingsford, C. (2011). A fast, lock-free approach for efficient parallel counting of occurrences of k-mers. Bioinformatics. 27, 764-770. doi: 10.1093/bioinformatics/btr011

Moriya, Y., Itoh, M., Okuda, S., Yoshizawa, A. C., and Kanehisa, M. (2007). KAAS: an automatic genome annotation and pathway reconstruction server. Nucleic Acids Res. 35, W182-W185. doi: 10.1093/nar/gkm321

Parra, G., Blanco, E., and Guigo, R. (2000). GeneID in Drosophila. Genome Res. 10, 511-515. doi: 10.1101/gr.10.4.511

Parra, G., Bradnam, K., and Korf, I. (2007). CEGMA: a pipeline to accurately annotate core genes in eukaryotic genornes. Bioinformatics. 23, 1061-1067. doi: 10.1093/bioinformatics/btm071

Peng, W., Xu, J., Zhang, Y., Feng, J., Dong, C., Jiang, L., et al. (2016). An ultra-high density linkage map and QTL mapping for sex and growth-related traits of common carp (Cyprinus carpio). Sci. Rep. 6:26693. doi: 10.1038/srep 26693

Price, A. L., Jones, N. C., and Pevzner, P. A. (2005). De novo identification of repeat families in large genomes. Bioinformatics. 21(Suppl. 1), i351-i358. doi: 10.1093/bioinformatics/bti1018

Quevillon, E., Silventoinen, V., Pillai, S., Harte, N., Mulder, N., Apweiler, R., et al. (2005). InterProScan: protein domains identifier. Nucleic Acids Res. 33, W116-W120. doi: 10.1093/nar/gki442

Shao, C., Li, C., Wang, N., Qin, Y., Xu, W., Liu, Q., et al. (2018). Chromosome-level genome assembly of the spotted sea bass, Lateolabrax maculatus. Gigascience. 7:giy114. doi: 10.1093/gigascience/giy114

Simao, F. A., Waterhouse, R. M., Ioannidis, P., Kriventseva, E. V., and Zdobnov, E. M. (2015). BUSCO: assessing genome assembly and annotation completeness with single-copy orthologs. Bioinformatics. 31, 3210-3212. doi: 10.1093/bioinformatics/btv351

Sola, L., Bressanello, S., Rossi, A. R., Iaselli, V., Crosetti, D., and Cataudella, S. (1993). A karyotype analysis of the genus dicentrarchus by different staining techniques. J. Fish Biol. 43, 329-337. doi: 10.1111/j.1095-8649. 1993.tb00567.x

Stanke, M., and Morgenstern, B. (2005). AUGUSTUS: a web server for gene prediction in eukaryotes that allows user-defined constraints. Nucleic Acids Res. 33, W465-W467. doi: 10.1093/nar/gki458

Tarailo-Graovac, M., and Chen, N. (2009). "Using RepeatMasker to identify repetitive elements in genomic sequences," in Current Protocols in Bioinformatics. ed B. D. Andreas. Wiley. doi: 10.1002/0471250953.bi0410s25

Tine, M., Kuhl, H., Gagnaire, P. A., Louro, B., Desmarais, E., Martins, R. S. T., et al. (2014). European sea bass genome and its variation provide insights into adaptation to euryhalinity and speciation. Nat. Commun. 5:5770. doi: $10.1038 /$ ncomms6770

Trapnell, C., Pachter, L., and Salzberg, S. L. (2009). TopHat: discovering splice junctions with RNA-Seq. Bioinformatics. 25, 1105-1111. doi: 10.1093/bioinformatics/btp120

Trapnell, C., Williams, B. A., Pertea, G., Mortazavi, A., Kwan, G., van Baren, M. J., et al. (2010). Transcript assembly and quantification by RNA-Seq reveals unannotated transcripts and isoform switching during cell differentiation. Nat. Biotechnol. 28, 511-515. doi: 10.1038/nbt.1621

Vij, S., Kuhl, H., Kuznetsova, I. S., Komissarov, A., Yurchenko, A. A., Van Heusden, P., et al. (2016). Chromosomal-level assembly of the Asian seabass genome using long sequence reads and multi-layered scaffolding. PLoS Genet. 12:e1005954. doi: 10.1371/journal.pgen.1005954.

Wang, C. M., Bai, Z. Y., He, X. P., Lin, G., Xia, J. H., Sun, F., et al. (2011). A high-resolution linkage map for comparative genome analysis and QTL fine mapping in Asian seabass, Lates calcarifer. BMC Genomics. 12:174. doi: 10.1186/1471-2164-12-174

Xu, Z., and Wang, H. (2007). LTR_FINDER: an efficient tool for the prediction of full-length LTR retrotransposons. Nucleic Acids Res. 35, W265-W268. doi: $10.1093 / \mathrm{nar} / \mathrm{gkm} 286$

Yokogawa, K., and Seki, S. (1995). Morphological and genetic-differences between Japanese and Chinese sea bass of the genus Lateolabrax. Jpn. J. Ichthyol. 41, 437-445.

Zhao, Y., Peng, W., Guo, H., Chen, B., Zhou, Z., Xu, J., et al. (2018). Population genomics reveals genetic divergence and adaptive differentiation of Chinese sea bass (Lateolabrax maculatus). Mar. Biotechnol. 20, 45-59. doi: $10.1007 /$ s10126-017-9786-0

Conflict of Interest Statement: The authors declare that the research was conducted in the absence of any commercial or financial relationships that could be construed as a potential conflict of interest.

The reviewer GW declared a past co-authorship with one of the authors YL to the handling editor.

Copyright (c) 2019 Chen, Li, Peng, Zhou, Shi, Pu, Luo, Chen and Xu. This is an open-access article distributed under the terms of the Creative Commons Attribution License (CC BY). The use, distribution or reproduction in other forums is permitted, provided the original author(s) and the copyright owner(s) are credited and that the original publication in this journal is cited, in accordance with accepted academic practice. No use, distribution or reproduction is permitted which does not comply with these terms. 